\title{
Mitral valve annulus and circumflex artery: In vivo study of anatomical zones
}

\author{
Vincenzo Caruso, MD, Usman Shah, FRCS (CTh), Haytham Sabry, FRCS (CTh), and \\ Inderpaul Birdi, FRCS (CTh)
}

\section{ABSTRACT}

Objective: To provide, with the use of preoperative coronary computed tomography angiography, an in vivo anatomical characterization of the relationship between the circumflex artery and mitral valve annulus to identify different risk classes and to increase the surgical awareness of those anatomical relations.

Methods: Ninety-five (mean age: $64.2 \pm 11.7$ ) consecutive patients, initially referred for elective minimally invasive mitral valve surgery, underwent preoperative coronary computed tomography angiography. The distance between the circumflex artery and mitral annulus was assessed using 6 points designed on the posterior mitral annulus, starting from the anterolateral to the posteromedial commissure; this design created an ideal 5-zone system. High-risk anatomy was defined as a distance less than $3 \mathrm{~mm}$ between the circumflex artery and the mitral valve annulus.

Results: The shortest distance between the circumflex artery and mitral valve annulus was observed at the area between the anterolateral commissure and the midpoint of $\mathrm{P} 1$ scallop, so-called zone $1(5.49 \pm 3.13 \mathrm{~mm})$, whereas the longest distance occurred at zone $5(12.03 \pm 4.93)$. Twenty-four patients $(25 \%)$ were identified with high-risk anatomy (mean distance $1.94 \pm 0.8 \mathrm{~mm}$ ). Left dominant and co-dominant hearts demonstrated a shorter circumflex artery-mitral valve annulus distance at all the zones. At multinomial logistic regression, the pattern of coronary dominance and the size of the circumflex artery were independent factors for high-risk anatomy.

Conclusions: Coronary computed tomography angiography is a useful investigation to identify patients at risk of circumflex artery flow disturbance; for high-risk anatomy, this knowledge may enhance a safer operative technique. (JTCVS Techniques 2020;4:122-9)

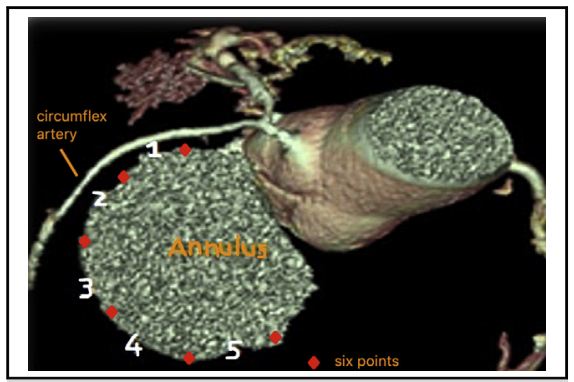

The "five zones" system designed along the posterior mitral leaflet.

CENTRAL MESSAGE

Coronary computed tomogra-

phy angiography gives informa-

tion about the distance between

the circumflex artery and the

mitral valve annulus, identifying

patients at high risk of circumflex

artery injury.

\section{PERSPECTIVE}

The consequence of circumflex artery injury during mitral valve surgery could be severe with important clinical implications. The knowledge of preoperative distance between the circumflex artery and the mitral valve annulus may help to identify patients at high risk of circumflex flow disturbance following mitral valve surgery.

See Commentaries on pages 130 and 132.

$\square$ Video clip is available online.

From the Cardiothoracic Department, Basildon and Thurrock University Hospitals NHS Foundation Trust, Basildon, United Kingdom.

Received for publication Aug 24, 2020; accepted for publication Sept 16, 2020; available ahead of print Sept 22, 2020.

Address for reprints: Vincenzo Caruso, MD, Basildon and Thurrock University Hospitals NHS Foundation Trust, Basildon SS16 5NL, United Kingdom (E-mail: vincenzocch@hotmail.it).

2666-2507

Copyright $@ 2020$ The Authors. Published by Elsevier Inc. on behalf of The American Association for Thoracic Surgery. This is an open access article under the CC BY-NCND license (http://creativecommons.org/licenses/by-nc-nd/4.0/).

https://doi.org/10.1016/j.xjtc.2020.09.013
Mitral valve surgery is known to be associated with the potential risk of disturbance of circumflex artery (CX) flow, as its course is intimately related to the mitral valve annulus (MVA) ${ }^{1-6}$ The true mechanism for this is still unclear, with authors suggesting direct suturing, ${ }^{3}$ laceration, or annuloplasty device distortion during mitral valve repair. The perioperative diagnosis can be extremely difficult to detect until the lateral wall injury to the left ventricle 


\section{Abbreviations and Acronyms \\ CCTA = coronary computed tomography angiography \\ $\mathrm{CX} \quad=$ circumflex artery \\ EuroSCORE II = European System for Cardiac Operative Risk Evaluation II \\ MVA = mitral valve annulus \\ OR $\quad=$ odds ratio \\ TOE $\quad=$ transesophageal \\ echocardiography}

becomes irreversible and is often realized only postoperatively as left ventricular dysfunction of unknown etiology.

Transesophageal echocardiography (TOE) has been used by experienced operators ${ }^{7}$ both in the preoperative and perioperative setting. It is a reliable technique to detect the distance between the CX and MVA, and it plays an important role in evaluating any wall motion abnormalities and the flow of the CX after mitral valve repair. However, this modality is operator dependent.

To understand the relationship between the $\mathrm{CX}$ and MVA, some authors have used multislice coronary computed tomography angiography (CCTA) preoperatively. ${ }^{8,9}$ In the present study, we describe our use of CCTA as an anatomical indicator of a high-risk CX-MVA relationship and the intraoperative modifications we have made to reduce any CX's flow disturbance.

\section{METHODS}

\section{Data Source}

A single-institution prospective cohort study was commenced to assess the relationship between CX and MVA in patients undergoing mitral valve surgery. Appropriate consent was obtained, before submission, for the publication of imagines and data.

Only those patients with severe mitral regurgitation and electively referred for minimally invasive surgery, under the care of a single surgeon, were enrolled in the study. Patients with major aortic surgery or urgent or emergency operation were excluded from the study. All patients underwent a preoperative study with CCTA (to assess the CX-MVA relationship and to assess the suitability for minimally invasive surgery) and coronary angiography (to assess the coronary anatomy and if associated lesions).

The final database was constituted by preoperative demographic variables, measurements of the CX-MVA distance, intraoperative interventional data, and postoperative outcomes.

\section{Coronary Computed Tomography Angiography}

A 64 slice multidetector CT (GE Lightspeed; GE Healthcare, Chicago, Ill) was used for scanning. A beta-blocker (metoprolol 25-50 mg) was administrated intravenously in patients with heart rates greater than 65 beats per minute. A prospective gated study was performed. An intravenous contrast material was injected at a rate of $4 \mathrm{~mL} / \mathrm{s}$, according to the patient's weight; data acquisition started after the threshold level of $+90-100$ Hounsfield units was reached, and it was performed during an inspiratory breathhold of around 8 seconds.

On end-diastolic phase, 2- and 4-chamber views were used to create a plane for the left ventricular short-axis, parallel to the mitral valve. This view was used to assess the mitral valve geometry: the intervalvular fibrosa, the 2 commissures, the entire mitral annulus, the anteroposterior diameter, and the intercommissural diameter.

Coronary arteries were reconstructed at $75 \%$ of the cardiac cycle; if the image quality was suboptimal, further reconstructions were taken at multiple different phases depending on whether a prospective or retrospective acquisition was performed. Reconstruction of the relationship of CX with MVA was performed using a GE workstation. Double oblique cross section allowed measurements to be taken at multiple different planes, and the shortest distance at each zone was recorded. Threedimensional reconstructions of MVA and CX were also acquired on selected cases.

CCTA-derived CX-MVA distance was measured over 5 zones lying between 6 points along the posterior mitral valve annulus, from commissure to commissure, in an anti-clockwise direction (Figure 1, insert): zone 1, between the anterolateral commissure and the midpoint of P1 scallop; zone 2, between the midpoint of $\mathrm{P} 1$ and $1 / 3$ of $\mathrm{P} 2$ scallop; zone 3, corresponding to the 2/3 remnant of P2 scallop; zone 4, from the insertion of P3 scallop to its midpoint; and zone 5, form the midpoint of $\mathrm{P} 3$ scallop to the posteromedial commissure (Figure 2). High-risk anatomy was arbitrarily defined as any zone where the CX-MVA distance was less than $3 \mathrm{~mm}$. Patients also underwent gated computed tomography scanning of the aorta to exclude atheroma, where minimally invasive surgery was confirmed as surgical approach. Preoperative 3-dimensional TOE and invasive coronary angiogram were performed in all patients and the different pattern of coronary dominance classified as per guidelines. ${ }^{10}$

\section{Intraoperative and Postoperative Course}

After induction of general anesthesia, 3-dimensional TOE analysis of the heart was undertaken, with special emphasis on elucidating the mitral valve apparatus, any regional wall motion of the left ventricle, and the $\mathrm{CX}$ flow. In $100 \%$ of cases, the CX flow was seen at zone 1 , and in $90 \%$ of case, the flow was seen as far as zone 3 . The technique for demonstration of the $\mathrm{CX}$ anatomy has been previously described. ${ }^{7,11}$

Following completion of the repair, the patient was weaned off from cardiopulmonary bypass. Three-dimensional TOE was performed to examine the adequacy of the repair, presence of new regional wall motion abnormalities, and to confirm a normal flow in the CX. New lateral wall ischemia was excluded by 12-lead electrocardiogram, whenever possible.

All patients underwent repeated transthoracic echocardiography before discharge and at 3 months postoperatively, to assess the result of the mitral valve repair and/or to evaluate the presence of new left ventricle wall motion abnormalities, perhaps indicative of CX flow disturbance.

\section{Statistical Analysis}

SPSS software, version 26.0 (IBM Corp, Armonk, NY), was used for statistical analysis. Continuous variables are presented as mean \pm standard deviation, and categorical variables are presented as absolute numbers and/or percentages. The Friedman test was used to assess statistically significant differences in the CX-MVA relationship within the different zones. Differences in the CX-MVA distance between the different coronary patterns and sex were assessed with a Kruskal-Wallis test and a Mann-Whitney $U$ test, respectively. Correlation between variables was conducted using bivariate correlation analysis. Binary and multilogistic regression was used to identify potential predictors between dependent and independent variables. For all tests, a $P$ value $<.05$ was considered statistically significant.

\section{RESULTS}

\section{Pathway Redesign}

Before the use of CCTA as preoperative tool, we experienced 2 cases of $\mathrm{CX}$ flow disturbance following minimally invasive mitral valve repair. The diagnosis 


\section{MITRAL VALVE ANNULUS AND CIRCUMFLEX ARTERY: IN VIVO STUDY OF DIFFERENT ANATOMICAL ZONES}

95 patients $\rightarrow$ Coronary Computed Tomography Angiography prior to mitral valve surgery

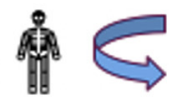

With the scan, six points were designated on the posterior mitral annulus This design created a FIVE ZONE SYSTEM

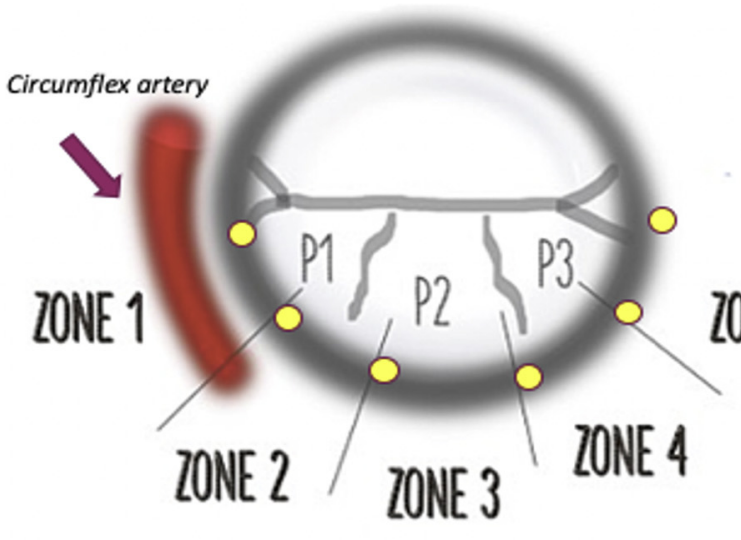

Acquisition of knowledge of distance (D) circumflex artery-mitral valve

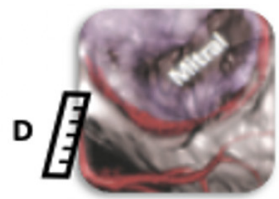

\begin{tabular}{|r|r|r|r|r|}
\hline ZONE 1 & ZONE 2 & ZONE 3 & ZONE4 & ZONE5 \\
\hline S.49 & 6.92 & 9.03 & 10.8 & 1203 \\
\hline
\end{tabular}

D<3mm $\rightarrow$ High Risk

24 patients (25\%)

Mean distance at

zone 1: $1.94 \pm 0.8 \mathrm{~mm}$

D $>3 \mathrm{~mm} \rightarrow$ Low Risk

71 patients (75\%).

Mean distance at zone 1: $6.7 \pm 2.6 \mathrm{~mm}$
Surgical Attention!!

Adjustment in surgical technique

FIGURE 1. From top to bottom and from left to right: methods and final cohort of the study; 5 zones identified on the posterior mitral annulus, lying between 6 points (yellow points); mean distance between the circumflex artery and the posterior mitral annulus; high- and low-risk anatomy identified in the study population; possible implications of the knowledge of high-risk anatomy.

was made postoperatively after episodes of electrical instability (sustained ventricular tachycardia and nonsustained ventricular polymorphic ectopics) with evidence of poor left ventricle ejection fraction. The coronary angiogram demonstrated, in both cases, occlusion of a dominant CX.

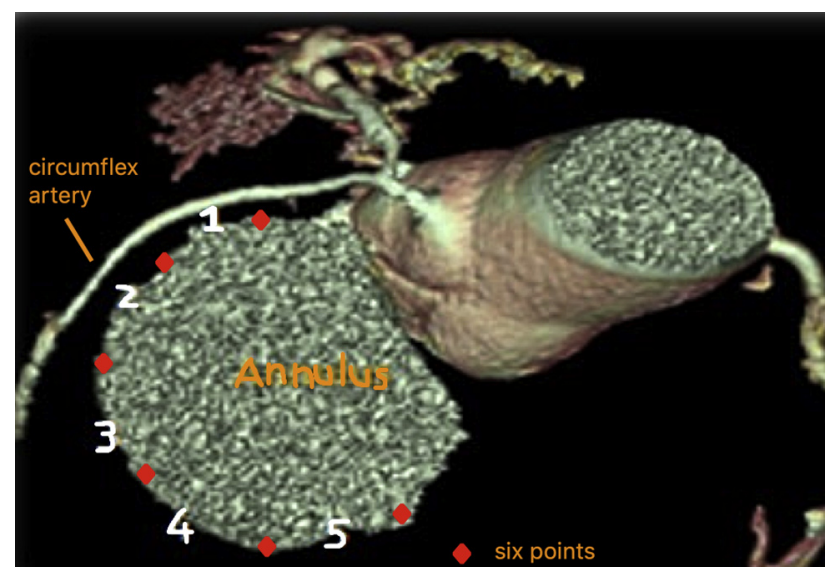

FIGURE 2. Coronary computed tomography angiography reconstruction: mitral valve plane with the 5 zones described along the posterior mitral leaflet, numbered from 1 to 5 , in anticlockwise direction. These zones are lying between 6 points (red squares). The circumflex artery (yellow arrow) lies close to the zone 1.
Both prestudy cases underwent CCTA analysis retrospectively and in each case, the CX was abutting the MVA at zone 1 and it would have been classified as high-risk anatomy in the present study (CX-MVA distance less than $3 \mathrm{~mm}$ ).

On the basis of this unusual experience in our practice, 95 patients underwent preoperative CCTA study of CX-MVA relationships. Baseline and perioperative characteristics were collected (Table 1).

\section{Preoperative Angiography}

Invasive coronary angiography revealed right dominance in 76 patients $(80 \%)$, left dominance in $9(9 \%)$, and codominance in 11 patients $(11 \%)$. The $\mathrm{CX}$ size was classified as small (diameter less than $3.4 \mathrm{~mm}$ ) in size in 26 patients (27\%), normal (diameter between 3.5 and $4.2 \mathrm{~mm}$ ) in 39 patients $(42 \%)$, and big (diameter more than $4.2 \mathrm{~mm}$ ) in the remnant 30 patients $(31 \%) .^{12}$

\section{CX-Annulus Relationship}

The shortest CX-MVA distance was at zone 1 $(5.49 \pm 3.13 \mathrm{~mm})$, whereas the longest distance occurred at zone 5 (12.03 \pm 4.93$)$ (Table 2). At univariate analysis, no significant differences were observed in regards of age, sex, or European System for Cardiac Operative Risk Evaluation II (EuroSCORE II) between the 3 different 
TABLE 1. Baseline characteristics, preoperative echocardiographic findings, and coronary dominance of the overall cohort

\begin{tabular}{lc}
\hline \multicolumn{1}{c}{ Overall cohort } & $\mathbf{n = 9 5}$ \\
\hline Age at surgery, y & $64.2 \pm 11.7$ \\
Sex, female (\%) & $30(31 \%)$ \\
\hline Reoperation & $5(5 \%)$ \\
\hline Logistic EuroSCORE & $3.07(\mathrm{IQR} 2.87)$ \\
Carpentier's class I & $10(11 \%)$ \\
Carpentier's class II & $54(57 \%)$ \\
Carpentier's class IIIa & $4(4 \%)$ \\
Carpentier's class IIIb & $27(28 \%)$ \\
Ejection fraction & $52.2 \pm 3.4$ \\
Right dominance & $76(80 \%)$ \\
\hline Left dominance & $9(9 \%)$ \\
Co-dominance & $10(11 \%)$ \\
\hline Low-risk anatomy & $71(75 \%)$ \\
High-risk anatomy & $24(25 \%)$ \\
\hline
\end{tabular}

EuroSCORE II, European System for Cardiac Operative Risk Evaluation II; IQR, interquartile range.

dominance patterns. However, there was statistically significant difference in regards of CX size (right dominance: small $34 \%$ [ $\mathrm{n}=26]$, normal $38 \%$ [n $=29$ ], big $28 \%$ $[\mathrm{n}=21]$; left dominance: small 0 [ $\mathrm{n}=0]$, normal $33 \%$ $[n=3]$, big $67 \%[n=6]$; codominance: small $0[n=0]$, normal $70 \%[\mathrm{n}=7]$, big $30 \%[\mathrm{n}=3] ; P=.013)$, and the different zones (zone 1: $P<.05$; zone $2: P<.01$; zone 3: $P<.01$; zone $4: P<.01$; zone 5: $P<.05$ ) (Table 3 ).

At a bivariate correlation test between the previous significant variables listed in Table 3, a significant statistically correlation was observed between the type of dominance and the CX size (rho $=0.265, P<.01$ ), zone 1 (rho $=0.241, P<.05)$, zone 2 (rho $=0.322, P<.01)$, zone 3 (rho $=0.342, P<.01$ ), zone 4 (rho $=0.352$, $P<.01)$ but not for zone 5 (rho $=0.134, P>.05)$ (Table 4$)$.

Furthermore, multinomial logistic regressions analysis was adjusted for only significant variables in the bivariate correlation. The regression analysis showed a statistical weight for independent predictor variables only for the CX size $\left(\chi^{2}=18.8, P<.001\right)$ and zone $1\left(\chi^{2}=6.99\right.$, odds ratio $[\mathrm{OR}], 3.8$ (confidence interval $[\mathrm{CI}], 1.1-12.8$ ), $P<.05)$ (Table 5).

Left dominant and co-dominant hearts demonstrated a shorter CX-MVA distance at all the zones from 1 to 5; the pairwise comparison of dominance showed that this was not statistically significant $(P>.05)$ for all the zones except for zone 1 , where a statistical difference was observed only for the left dominant heart (left-right dominance: $P<.05$; co-dominance-right dominance: $P>.05$ ).

No significant differences were observed between CXMVA distance and sex at all zones $(P>.05$, zone 1: OR, 1.16 [CI, 0.42-3.2], $P>.05$; zone 2: OR, 0.8 [CI, 0.242.6], $P>.05$; zone 3: OR, 0.45 [CI, 0.27-7.5], $P>.05$; zone 4: OR, 1.4 [CI, 1.2-1.7], $P>.05$; zone 5: OR, 1.4 [CI, 1.2-1.7], $P>$.05) (Table 6).

\section{Surgical Technique}

As result of the preoperative investigations, 10 patients (11\%) underwent a traditional sternotomy approach; the remaining patients $(89 \%)$ underwent right anterior thoracotomy. Sternotomy was performed in 3 patients for concomitant procedure (tricuspid valve repair or coronary artery bypass graft) and in 7 patients because of the shorter distance of CX-MVA (zone 1 and/or zone 2). In the minimally invasive group, an endo-aortic balloon occlusion device was deployed in 10 patients and an external aortic occlusion device was used in 73 patients. In 3 patients, conversion from mini-thoracotomy to median sternotomy was decided intraoperatively (failure to deploy the endoclamp safely [ $n=1]$, rapid onset of ventricular fibrillation postoperatively due to massive occult left side pneumothorax $[\mathrm{n}=1]$, or electrocardiographic signs of myocardial ischemia intraoperatively $[\mathrm{n}=1]$ ).

The mitral valve was repaired in $93 \%$ of the cases $(\mathrm{n}=88)$ with the implant of an annuloplasty device (Table 7); the repair was completed with chordal reconstruction in 60 patients $(63 \%)$ and posterior leaflet resection in 1 patient $(1 \%)$.

For 7 patients ( $7 \%$ ), it was necessary to replace the mitral valve; in this group, the CX-MVA distance was always more than $3 \mathrm{~mm}$ and the patients considered as having low-risk anatomy.

TABLE 2. Mean distance ( $\mathrm{mm}$ ) between the circumflex artery and the mitral annulus, at 5 zones

\begin{tabular}{lccccc}
\hline \multicolumn{1}{c}{ Overall cohort } & Zone 1 & Zone 2 & Zone 3 & Zone 4 & Zone 5 \\
\hline Mean & 5.49 & 6.92 & 9.07 & 10.8 & 12.03 \\
SD & 3.13 & 3.39 & 3.75 & 5.22 & 4.93 \\
Median & 4.8 & 6.6 & 9 & 10.6 & 12 \\
Minimum value & 0.60 & 1 & 2.9 & 1.9 & 25 \\
Maximum value & 12 & $4.9 \pm 2.6$ & $7.7 \pm 3.1$ & $9.2 \pm 5$ & $11.3 \pm 5.1$ \\
Low risk, mean \pm SD & $6.7 \pm 2.6$ & $7.5 \pm 3.3$ & $9.5 \pm 3.8$ & $10.6 \pm 6$ \\
\hline High risk, mean \pm SD & $1.94 \pm 0.8$ & & & $12.5 \pm 4.3$ \\
\hline
\end{tabular}

SD, Standard deviation. 
TABLE 3. Univariate analysis: dominance type and covariates

\begin{tabular}{|c|c|c|c|c|}
\hline Variables & Right dominance $(n=76,80 \%)$ & Left dominance $(n=9,9.5 \%)$ & Co-dominance $(n=10,10.5 \%)$ & $P$ value \\
\hline Age, y mean \pm SD & $64.5 \pm 12.3$ & $66.6 \pm 7.5$ & $59.6 \pm 7.6$ & .371 \\
\hline Sex & & & & .165 \\
\hline Male & $52(68)$ & $8(89)$ & $5(50)$ & \\
\hline Female & $24(32)$ & $1(11)$ & $5(50)$ & \\
\hline EuroSCORE II, median (IQR) & $3.1(2-5.7)$ & $3(2-4)$ & $1.8(1.5-4)$ & .374 \\
\hline Circumflex size, n (\%) & & & & $.013^{*}$ \\
\hline Small & $26(34)$ & $0(0)$ & $0(0)$ & \\
\hline Normal & $29(38)$ & $3(33)$ & $7(70)$ & \\
\hline Big & $21(28)$ & $6(67)$ & $3(30)$ & \\
\hline \multicolumn{5}{|l|}{ Zone, mean \pm SD } \\
\hline Zone 1 & $5.9 \pm 3.2$ & $3 \pm 2.1$ & $4.6 \pm 2.3$ & $.021 *$ \\
\hline Zone 2 & $7.5 \pm 3.4$ & $4.5 \pm 1.7$ & $4.9 \pm 2.7$ & $.006 \dagger$ \\
\hline Zone 3 & $9.7 \pm 3.8$ & $6.5 \pm 1.3$ & $6.6 \pm 3.2$ & $.004 \dagger$ \\
\hline Zone 4 & $11.7 \pm 5.2$ & $6.9 \pm 3$ & $7.8 \pm 4$ & $.004 \dagger$ \\
\hline Zone 5 & $12.4 \pm 4.6$ & $8 \pm 5.7$ & $12.6 \pm 5$ & $.034 *$ \\
\hline
\end{tabular}

$S D$, Standard deviation; EuroSCORE II, European System for Cardiac Operative Risk Evaluation II; IQR, interquartile range. $* P<.05$. $\dagger P<.01$.

In the overall cohort, there were no deaths at the mean follow-up (11.5 \pm 4.3 months); all patients underwent transthoracic echocardiography that showed null (low risk: $\mathrm{n}=65[92 \%]$; high risk: $\mathrm{n}=22[92 \%])$ or mild (low-risk: $\mathrm{n}=5[7 \%]$; high risk: $\mathrm{n}=2[8 \%]$ ) residual mitral regurgitation in all the patients, except for 1 who underwent redo mitral valve surgery for infective endocarditis (Table 8).

\section{Low-Risk Anatomy and Surgical Management}

Seventy-one patients $(75 \%)$ showed a CX-MVA distance greater than $3.0 \mathrm{~mm}$ at all zones; the mean distance at zone 1 was $6.7 \pm 2.6 \mathrm{~mm}$. Seven patients $(8 \%)$ had valve replacement (tissue valve: $\mathrm{n}=4$ [57\%], mechanical valve: $\mathrm{n}=3$

TABLE 4. Bivariate correlation, overall cohort: Spearman rankorder correlation for dominance type and correlated variables

\begin{tabular}{lc}
\hline \multicolumn{1}{c}{ Variable } & $\begin{array}{c}\text { Correlation } \\
\text { coefficient }- \text { rho } \boldsymbol{P} \text { value }\end{array}$ \\
\hline Group & 1 \\
Zone 1 & .241 \\
& $.019 *$ \\
Zone 2 & 0.322 \\
& $0.001 \dagger$ \\
Zone 3 & 0.342 \\
& $0.001 \dagger$ \\
Zone 4 & 0.351 \\
& $0.001 \dagger$ \\
Zone 5 & 0.134 \\
& 0.195 \\
Circumflex size & 0.265 \\
& $0.009 \dagger$ \\
\hline
\end{tabular}

$* P<.05 . \dagger P<.01$.

[43\%]). When the valve was repaired, flexible bands $(\mathrm{n}=32,45 \%)$, undersized rigid ring $(\mathrm{n}=18,25 \%)$, or semi-rigid ring $(\mathrm{n}=15,21 \%)$ were used.

None of these patients had intraoperative or early postoperative signs of myocardial ischemia; in 2 of those patients, a minimal reduction of the left ventricle ejection fraction was observed on the predischarge transthoracic echocardiography ( $35 \%$ and $38 \%$, respectively), but, at 3-month follow-up, this had returned to preoperative levels. At mean follow-up, the mean left ventricle ejection fraction for the low-risk group was $50.2 \pm 7 \%$.

\section{High-Risk Anatomy and Surgical Management}

In 24 patients, the mean distance between CX-MVA was $1.94 \pm 0.8 \mathrm{~mm}$ at zone 1 ; interestingly, a left dominance was observed only in 5 patients, co-dominance in 1 case, and right dominance was seen in the remaining 16 cases.

Depending on the mechanism of mitral regurgitation, the repair was always completed with the implant of an annuloplasty device: flexible band $(\mathrm{n}=12,50 \%)$; undersized rigid ring $(\mathrm{n}=9,38 \%)$; or semi-rigid ring $(\mathrm{n}=2,8 \%)$.

TABLE 5. Multinomial logistic regression: dominance and high riskanatomy (distance circumflex artery-mitral valve annulus $<\mathbf{3 ~ m m}$ )

\begin{tabular}{lcc}
\hline \multicolumn{1}{c}{ Variable } & $\boldsymbol{\chi}^{\mathbf{2}}$ & $\begin{array}{c}\text { Independent predictor } \boldsymbol{P} \\
\text { value }\end{array}$ \\
\hline Zone 1 & 6.99 & $.030^{*}$ \\
Zone 2 & 0.599 & .741 \\
Zone 3 & 3.2 & .200 \\
Zone 4 & 3.7 & .157 \\
Zone 5 & 2.5 & .380 \\
Circumflex size & 18.8 & $.001 \dagger$ \\
$* P<.05 . \dagger P<.01$. & &
\end{tabular}


TABLE 6. Risk factor for high risk anatomy

\begin{tabular}{lcccc}
\hline \multicolumn{1}{c}{ Risk factor } & N high-risk $(\%)$ & OR $(\mathbf{9 5} \% \mathbf{C I})$ & $\chi^{2}$ & $\boldsymbol{P}$ value \\
\hline Sex (female) & & & & \\
Zone 1 & $24(25.3)$ & $1.16(0.42-3.2)$ & 0.086 & .490 \\
Zone 2 & $14(14.7)$ & $0.8(0.24-2.6)$ & 0.130 & .469 \\
Zone 3 & $2(2.1)$ & $0.45(0.27-7.5)$ & 0.321 & .534 \\
Zone 4 & $4(4.2)$ & $1.4(1.2-1.7)$ & 1.92 & .213 \\
Zone 5 & $3(3.2)$ & $1.4(1.2-1.7)$ & 1.43 & .316 \\
Dominance & & & & \\
$\quad$ left and & & & & \\
$\quad$ codominance) & & & & \\
Zone 1 & $21(24.7)$ & $3.8(1.1-12.8)$ & 5.15 & $.037 *$ \\
Zone 2 & $11(12.9)$ & $1.9(0.4-8.09)$ & 0.77 & .329 \\
Zone 3 & $1(1.2)$ & $1.1(1-1.4)$ & 0.12 & .894 \\
Zone 4 & $3(3.5)$ & $2.8(2.6-22.7)$ & 10.3 & .290 \\
Zone 5 & $3(3.5)$ & $3.6(6.3-29.5)$ & 26.2 & .100 \\
\hline OR, Odds ratio; $C I$, confidence interval. $* P<.05$. & &
\end{tabular}

In 14 cases $(58 \%)$, with the knowledge of a closer distance CX-MVA, no stiches were passed in any zones identified as a high risk (zone 1 in all of those cases, zone 1 and 2 in 1 case); for all these cases, the reparative gesture was initially performed with implant of neo-chordae for the prolapsing scallop.

Despite these changes in the surgical technique, the intraoperative TOE showed a good reparative result (null residual mitral regurgitation in all cases); these results were confirmed at postoperative echocardiography. At the mean follow-up, the mean ejection fraction, for the high-risk group, was of $51.2 \pm 5 \%$ (Table 8 ).

Only one patient experienced intraoperative CX obstruction: this has been already described in a case report by the same authors. The early diagnosis and recognition permitted a prompt treatment and resolution of the CX obstruction. $^{13}$

\section{DISCUSSION}

CX injury is thought to be a rare complication of mitral valve surgery, with an incidence from between $0.3 \%$ and

TABLE 7. Annuloplasty device used

\begin{tabular}{lc}
\hline \multicolumn{1}{c}{ Type } & n (\%) \\
\hline Ring & $44(46)$ \\
$\quad$ Carpentier-McCarthy-Adams IMR Etlogix ring & $27(61)$ \\
$\quad$ (Edwards Lifescience, Irvine, Calif) & \\
$\quad$ Carpentier Edwards Physio II annuloplasty ring & $17(39)$ \\
$\quad$ (Edwards Lifescience) & \\
Band & $44(46)$ \\
Duran AnCore Band, (Medtronic, Minneapolis, Minn) & $18(40)$ \\
CG Future Band (Medtronic) & $26(60)$ \\
Valve replacement & $7(7)$ \\
$\quad$ Hancock II (Medtronic) & $4(57)$ \\
St Jude Master (St Jude Medical, St Paul, Minn) & $3(43)$ \\
\hline
\end{tabular}

TABLE 8. Postoperative echocardiographic data: mean comparison with Kruskal-Wallis test

\begin{tabular}{|c|c|c|c|}
\hline Variables & $\begin{array}{c}\text { Low risk } \\
(\mathrm{n}=\mathbf{7 1}, \mathbf{7 5} \%)\end{array}$ & $\begin{array}{c}\text { High risk } \\
(n=24,25 \%)\end{array}$ & $P$ value \\
\hline Ejection fraction, $\%$ & $50.2 \pm 7$ & $51.2 \pm 5$ & .266 \\
\hline LVEDD, mm & $38.5 \pm 4.7$ & $39 \pm 5.2$ & .119 \\
\hline LVESD, mm & $22.8 \pm 10.7$ & $24.5 \pm 11$ & .658 \\
\hline TAPSE, mm & $8.8 \pm 5$ & $9 \pm 5.1$ & .059 \\
\hline Residual MR & & & .374 \\
\hline Null & $65(92)$ & $22(92)^{*}$ & \\
\hline Mild & $5(7)$ & $2(8)$ & \\
\hline Moderate & $0(0)$ & $0(0)$ & \\
\hline Severe & $1(1) \dagger$ & $0(0)$ & \\
\hline
\end{tabular}

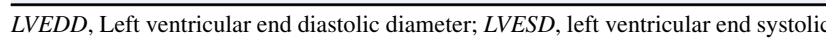
diameter; TAPSE, tricuspid annular plane systolic excursion; $M R$, mitral regurgitation. *In 24 patients, no stiches were passed at zone 1 (see text). †Mitral valve endocarditis at 2 months' follow-up.

$1.5 \% .^{1-6}$ In a recent study of 1705 patients undergoing mitral valve repair with ejection fraction assessment of greater than $60 \%$ preoperatively, $18 \%(n=314)$ of these patients were found to have ejection fraction less than $50 \%$ immediately postoperatively. Only one third of these patients improved to preoperative levels at intermediateterm follow-up, suggesting that at least $12 \%$ of patients with good left ventricular function preoperatively will suffer reduced function postoperatively. ${ }^{14}$

No study to date has examined the causes of this dysfunction; this is very likely to be, at least in part, related to CX flow disturbances. The consequences of $\mathrm{CX}$ flow disturbances can be significant and with potentially devastating effects as in the 2 pre-study cases mentioned in this article and occurred in our unit before the start of this study with CCTA.

Intraoperative TOE is a helpful tool, providing an early identification of any abnormalities on the regional wall motion and the ventricular function; we also used a color Doppler analysis of the CX flow during and after the mitral repair. However, this modality is operator dependent. Ender and colleagues ${ }^{7}$ describe an eloquent method for perioperative TOE evaluation of CX artery integrity during mitral valve surgery. We employed their approach and despite considerable experience in this area, we were not always able to fully visualize the $\mathrm{CX}$ throughout its posterior annular course.

For this reason, we chose to employ the use of preoperative CCTA to describe the CX-MVA relationship using a "five-zone system" (Video 1). Other authors have used CCTA in a similar way, and the results are in concordance with respect to identification of zone 1 as the closest area of CX-MVA distance. ${ }^{15} \mathrm{We}$ also found that the least zone of variability was in this area, which is important as it lends credence to the accuracy of CCTA in identifying the 


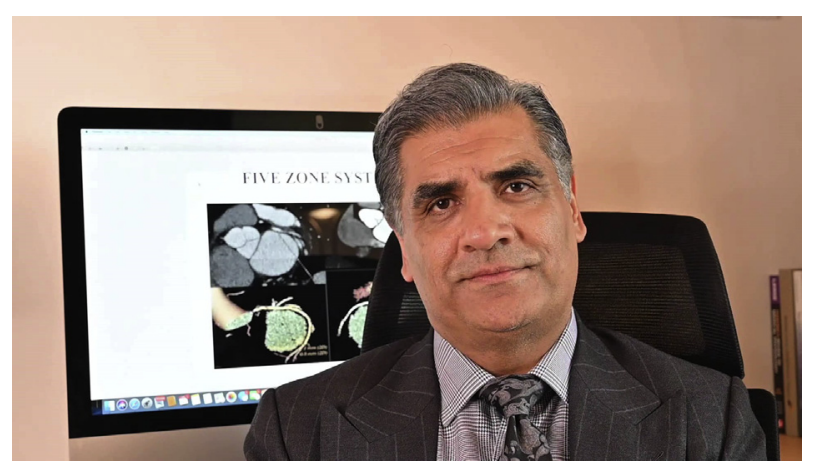

VIDEO 1. Author I. Birdi describes the 5-zone system and the rationale of the use of coronary computed tomography angiography to better understand the relationship between mitral valve annulus and circumflex artery. He then describes the case of a patient, with high-risk anatomy, who had intra-operative circumflex artery injury; this required return in theatre to remove the first annuloplasty device implanted and to perform a coronary artery by-pass graft on the region of the circumflex area. Video available at: https://www.jtcvs.org/article/S2666-2507(20)30516-2/fulltext.

greatest area of risk. Our data also concord with anatomical and clinical data from other studies ${ }^{16-20}$ in regard to the importance of zone 1; this was the only predictor risk for high-risk anatomy. Our experience with CCTA was that it was very reproducible, and we were able to confidently identify high- and low-risk anatomies. As noted, both our pre-study cases with CX-injury underwent computed tomography analysis retrospectively; in both cases, the CX abutted the MVA at zone 1.

In regard of coronary dominance pattern, previous studies have debated whether it is related to CX injury; Virmani and colleagues ${ }^{6}$ first reported that the greater risk of iatrogenic CX injury occurs in patients with left dominant or codominant coronary circulation; these findings were later confirmed by Kaklikkaya and Yeginoglu, ${ }^{21}$ who reported that the shorter distance was in left dominant hearts and that the proximal one-third of CX was the greatest area of risk. In contrast with these studies, a report by Pessa and colleagues ${ }^{18}$ suggested that the incidence of CX injury was independent of coronary dominance: right dominance heart had a minimum distance of $1.01 \mathrm{~mm}$ between CX-MVA.

In our study, left dominance and right dominance were the prevailing systems in the high-risk anatomy group, with no significant statistical differences, expect for zone 1. Thus, we believe that dominance, per se, is not a reliable measure of risk. Neither sex was found to be a predictive risk factor for high-risk anatomy; age and EuroSCORE II were also not predictive for high-risk anatomy:

Regarding the surgical technique, in cases of closer CXMVA relationships, we have maintained consistent diligence during suture placement, directing the needle away from the annulus at all times. With the CCTA data, we enforced the concept that the proximity of the CX to the MVA, as well as the length of its course within the atrioventricular groove, rendered it susceptible to distortion or compression; in this contest, sutures and inserted annuloplasty devices may play a role, but this concept is limited to our small series experience.

In more than one half of high-risk anatomy group, suture avoidance at high-risk zones was performed; whilst it is possible that this may have helped to mitigate CX flow disturbance, suture avoidance at zone 1 in our study case of CX disturbance did not avoid the complication. ${ }^{13}$ Nevertheless, we did not see any reduction in mitral valve competence by observing this approach and for now it remains our practice.

Interestingly, in the present manuscript, the one incidence of CX injury in the study group and both cases in the pre-study period all received semi-rigid rings. We continue to believe that the use of semi-rigid annuloplasty devices offer the best long-term stability following mitral valve repair, but we found that a flexible device may provide similar reparative outcomes. Silberman and colleagues ${ }^{22}$ showed that clinical and hemodynamic results were better with rigid mitral annuloplasty rings compared with flexible rings although this study considered only patient with ischemic MR. In contrast, $\mathrm{Hu}$ and $\mathrm{Zhao}^{23}$ report no significant difference in overall survival, regurgitation recurrence, and left ventricular performance between the flexible and rigid rings. As this debate is certain to continue, we believe that CCTA allows us to select those cases where the use of a flexible devise is safe and may provide a satisfactory repair.

In addition, the identification of high-risk anatomy preoperatively with CCTA may warrant an early on-table angiography; in selected cases, the mitral valve repair is performed now, in our unit, in the hybrid theater, with an angiographic control of the integrity of the CX after weaning off the cardiopulmonary bypass.

\section{Limitations}

The small number of patients enrolled in this study limits our understanding of the effects of coronary dominance and circumflex proximity. We were unable to make prospective comparisons between CCTA findings and TOE data, as our ability to visualize the $\mathrm{CX}$ throughout its course was not always possible. This may be a reason to support the use of CCTA.

\section{CONCLUSIONS}

Preoperative assessment of circumflex proximity to the mitral valve annulus with CCTA is of great value in identifying patients at high risk of circumflex flow disturbance following mitral valve surgery. Zone 1 is the area of greatest risk; no relationships are observed with coronary artery dominance, age, sex, or EuroSCORE II. 
This knowledge may enhance a safer operative technique and may increase attention regarding postoperative subtle signs of myocardial ischemia. The immediate and prompt recognition and treatment of this complication is mandatory to avoid any dramatic complications.

\section{Conflict of Interest Statement}

I.B. has a patent for a prosthetic device for mitral repair, issued in United Kingdom, pending in the European Union, broadly relevant to the work. All other authors reported no conflicts of interest.

The Journal policy requires editors and reviewers to disclose conflicts of interest and to decline handling or reviewing manuscripts for which they may have a conflict of interest. The editors and reviewers of this article have no conflicts of interest.

\section{References}

1. Aybek T, Risteski P, Miskovic A, Simon A, Dogan S, Moritz A. Seven years' experience with suture annuloplasty for mitral valve repair. J Thorac Cardiovasc Surg. 2006;131:99-106

2. Meursing DF, Boonswang NA, Dobrilovic N, Wait MA. Perioperative myocardial infarction secondary to dynamic circumflex coronary artery occlusion after mitral valve repair. Tex Heart Inst J. 2006;33:85-7.

3. Mulpur AK, Kotidis KN, Nair UR. Partial circumflex artery injury during mitral valve replacement: late presentation. J Cardiovasc Surg. 2000;41:333-4.

4. Tavilla G, Pacini D. Damage to the circumflex coronary artery during mitral valve repair with sliding leaflet technique. Ann Thorac Surg. 1998;66:2091-3.

5. Danielson GK, Cooper E, Tweedore DN. Circumflex coronary artery injury during mitral valve replacement. Ann Thorac Surg. 1967;4:53-9.

6. Virmani R, Chun PK, Parker J, McAllister HA Jr. Suture obliteration of the circumflex coronary artery in three patients undergoing mitral valve operation. Role of left dominant or codominant coronary artery. $J$ Thorac Cardiovasc Surg. 1982;84:773-8.

7. Ender J, Selbach M, Borger MA, Krohmer E, Falk V, Kaisers U, et al. Echocardiographic identification of iatrogenic injury of the circumflex artery during minimally invasive mitral valve repair. Ann Thorac Surg. 2010;89:1866-72.

8. Choure A, Garcia MJ, Hesse B, Sevensma M, Maly G, Greenberg NL, et al. In vivo analysis of the anatomical relationship of coronary sinus to mitral annulus and left circumflex coronary artery using cardiac multidetector computed tomography. J Am Coll Cardiol. 2006;48:1938-45.

9. Delgado V, Tops LF, Schuijf JD, de Roos A, Brugada J, Schalij MJ, et al. Assessment of mitral valve anatomy and geometry with multislice computed tomography. J Am Coll Cardiol Imaging. 2009;2:556-65.

10. Scanlon PJ, Faxon DP, Audet AM, Carabello B, Dehmer GJ, Eagle KA, et al. ACC/AHA guidelines for coronary angiography: a report of the American
College of Cardiology/American Heart Association task force on practice guidelines (committee on coronary angiography) developed in collaboration with the Society for Cardiac Angiography and Interventions. J Am Coll Cardiol. 1999;33: 1756-824.

11. Nakajima H, Ikari Y, Kigawa I, Kitamura T, Hatori M, Tooda E, et al. Rapid diag nosis and management of intraoperative myocardial infarction during valvular surgery: using intraoperative transesophageal echocardiography followed by emergency coronary artery bypass grafting without coronary angiography. Echocardiography. 2005;22:834-8.

12. Dodge JT Jr, Brown BG, Bolson EL, Dodge HT. Lumen diameter of normal human coronary arteries. Influence of age, sex, anatomic variation, and left ventricular hypertrophy or dilation. Circulation. 1992;86:232-46.

13. Caruso V, Sabry H, Birdi I. Dramatic resolution of an immediate postoperative distortion of the circumflex artery during mitral valve surgery. J Card Surg. 2020:35:1135-7.

14. Quintana E, Suri RM, Thalji NM, Daly RC, Dearani JA, Burkhart HM, et al. Lef ventricular dysfunction after mitral valve repair-the fallacy of "normal" preoperative myocardial function. J Thorac Cardiovasc Surg. 2014;148:2752-62.

15. Ghersin N, Abadi S, Sabbag A, Lamash Y, Anderson RH, Wolfson H, et al, The three-dimensional geometric relationship between the mitral valvar annulus and the coronary arteries as seen from the perspective of the cardiac surgeon using cardiac computed tomography. Eur J Cardiothorac Surg. 2013; 44:1123-30.

16. Cornu E, LacroixPH, Vhristides C, Laskar M. Coronary artery damage during mitral valve replacement. J Cardiovasc Surg. 1995;36:261-4.

17. Eto M, Morita S, Nakashima Y, Nishimura Y, Tominaga R. Morphometric study of the human mitral annulus: guide for mitral valve surgery. Asian Cardiovasc Thorac Ann. 2014:22:787-93.

18. Pessa CJN, Gomees WJ, Catani R, Prates JC, Buffolo E. Anatomical relationship between the posterior mitral valve annulus and the coronary arteries. Implications to operative treatment. Braz J Cardiovasc Surg. 2004;19:372-7.

19. Mantilla R, Legarra J, Pradas G, Bravo M, Sanmartin M, Goicolea J. Percutaneous coronary Intervention for iatrogenic occlusion of the circumflex artery after mitral annuloplasty. Revista Espanola de Cardiologia. 2004;57: $702-4$.

20. Aubert S, Barthelemy O, Landi M, Acar C. Circumflex coronary artery injury following mitral annuloplasty treated by emergency angioplasty. Eur J Cardiothorac Surg. 2008;34:922-4.

21. Kaklikkaya I, Yeginoglu G. Damage to coronary arteries during mitral valve surgery. Heart Surg Forum. 2003;6:E138-42.

22. Silberman S, Klutstein MW, Sabag T, Oren A, Fink D, Merin O, et al. Repair of ischemic mitral regurgitation: comparison between flexible and rigid annuloplasty rings. Ann Thorac Surg. 2009;87:1721-7.

23. Hu X, Zhao Q. Systematic evaluation of the flexible and rigid annuloplasty ring after mitral valve repair for mitral regurgitation. Eur J Cardiothorac Surg. 2011; 40:480-7.

Key Words: mitral anatomy, mitral murgery, circumflex artery, minimally invasive surgery, coronary computed tomography angiography 